\title{
Melt processable homo- and copolyimides with high thermo-oxidative stability as derived from mixed thioetherdiphthalic anhydride isomers
}

\author{
Y. Han, X. Z. Fang*, X. X. Zuo \\ Ningbo Key Laboratory of Polymer Materials, Polymers and Composites Division, Ningbo Institute of Materials \\ Technology \& Engineering, Chinese Academy of Sciences, Ningbo, 315201, P. R. China
}

Received 7 May 2010; accepted in revised form 15 July 2010

\begin{abstract}
A series of homo- and copolyimides based on mixed thioetherdiphthalic anhydride isomers (mixed-TDPA) were synthesized with several kinds of aromatic diamines. The properties of these polyimides were characterized by glass transition temperature $\left(T_{g}\right)$, thermal decomposition temperature, and melt processability. A series of copolyimides were prepared to achieve high $T_{g}$ concurrently with melt processability by means of selecting appropriate diamines and their composition in the copolyimides. As a result, we obtained rheological information for a series of polyimide resins as a function of temperature, time and shear rate. It is found that the processability (e.g., melt viscosity of polyimides) and ultimate product properties (e.g., $T_{g}$ of polyimides) can be systematically varied by changing the variety and composition of the aromatic diamines. It has been demonstrated that the incorporation of meta- or flexible diamines improve the melt processability of polyimides significantly. Meanwhile, $T_{g}$ of copolyimides from dual-diamines can be predicted and regulated. Accordingly, copolyimides from 1,3-phenylenediamine ( $m$-PDA) and 3,4'-oxydianiline (3,4'-ODA) were obtained with higher $T_{g}$ and lower melt viscosity. Such correlations of chemical structures and rheological behavior provide the necessary database for tailor-making new polyimide systems with desirable processability and physical properties.
\end{abstract}

Keywords: thermal properties, melt processable, isomeric polyimide, rheology

\section{Introduction}

Aromatic polyimides (PIs), due to their excellent thermal stability, superior mechanical strength and good chemical resistance properties, found their application in many industrial fields [1]. The outstanding properties of PIs are based on the combination of their stiff chain structures and ordered structure formation caused by strong interchain interactions. However, these advantages, meanwhile, give rise to poor solubility and processability of PIs. General methods to improve the processability are to incorporate flexible linkage into the polymer backbones [2-4]. This approach, nevertheless, inevitably leads to a decrease of glass transi- tion temperature $\left(T_{g}\right)$ in many cases. For instance, commercial thermoplastic polyimide Ultem possesses excellent melt processability but its longterm service temperature is limited to lower than $170^{\circ} \mathrm{C}$. Moreover, the thermoxidative stability of Ultem is also relatively poor because of the presence of thermally unstable isopropylidene groups in the polymer backbones [5].

Thermoplastic PIs are commonly applied in various industries because of the ease of processing, handling, and storage. Most application requires thermoplastic PIs to exhibit enhanced melt flow behavior at relatively low processing temperatures. However, the high melt viscosity of these materials 
requires high pressure to be used in processing to fabricate structural composites and adhesive joints. Therefore, improvements are required to reduce the melt viscosities without compromising other properties.

It was reasoned that introduction of the monomers containing asymmetric or noncoplanar structure to replace the linear symmetrical dianhydride in polymer chain could weaken the interchain interaction thus enhancing the solubility and melt flowability [6]. As of result of that, it has been further demonstrated that replacement of the symmetrical dianhydride in PIs with asymmetric ones decreases the melt viscosity but increases the $T_{g}$ relative to the corresponding symmetrical PIs [4, 7-9]. Thioetherdiphthalic anhydride (TDPA) is an aromatic bridged dianhydride which has three isomers as shown in Figure 1. The flexible thioether moiety offers the polymer with flexibility and easy melt processability. As a consequence, thioether links have been introduced to many polymers. And polythioetherimides have been synthesized by many researchers for different purposes [10-12]. In previous research, PIs based on 2,3,3',4'-thioetherdiphthalic anhydride (3,4'-TDPA) exhibited a higher $T_{g}$ than that of symmetric 3,3',4,4'-thioetherdiphthalic anhydride (4,4'-TDPA) and also presented a remarkable drop in the complex viscosity above $T_{g}$ [13]. This is attributed to the remarkable decrease in the intermolecular interactions of the geometrically irregular 3,4'-TDPA-based PI. According to the research on a similar dianhydride ODPA, TDPA-based polyimides show similar thermal stability and mechanical properties as ODPA-based polyimides, meanwhile, more excellent melt flowability. Moreover, the synthesis of TDPA is more convenient than that of ODPA [4].

Copolymerization by incorporating monomers with flexible moieties, which disrupts the regularity of molecular chain and prevents the interaction of polymer chains, is a simple but effective way for structure modification to balance the processability and the desired properties $[14,15]$. For example, 3,4'- oxydianiline (3,4'-ODA) has been proved to be a diamine with polyimide derivatives having better melt flowability than polymers from its isomer 4,4'-oxydianiline(4,4'-ODA) [16]. Other isomeric diamines, such as 1,3-bis(4-aminophenoxy) benzene (TPER) and 1,3-phenylenediamine ( $m$-PDA), can also offer polyimides relatively lower melt viscosity than the corresponding para-isomers [5, 17]. As a consequence, the processability of copolyimides is enhanced dramatically. The $T_{g}$ of these copolyimides generally range between that of homopolyimides from the two dianhydrides or diamines $[5,16,18]$. This principle makes it possible to predict the $T_{g}$ of copolyimides by appropriately selecting the structure and ratios of dianhydride and diamine component while obtaining improved melt flowability of PIs.

Based on the thermal and rheological behavior of 3,4'-TDPA based polyimides, we have successfully prepared copolyimides from mixture of three TDPA isomers (mixed-TDPA) [19]. These kinds of isomeric copolyimides show excellent melt processability similar to the homopolyimide from 3,4'TDPA. Even though PIs from mixed-TDPA didn't show obviously better melt flowability than that from 3,4'-TDPA, the mixed isomeric dianhydrides can be synthesized by a more convenient routine than that of 3,4'-TDPA [20], which is favorable to prepare a novel melt processable PI with relatively low cost. Meanwhile, it has also been proved that regulating the number-average molecular weight at an appropriate level by stoichiometric imbalance has a significant effect on the melt flowability and the $T_{g}$ of the copolyimides.

We wish to lower the viscosity of PIs by the replacement of the rigid repeat unit, namely 4,4'-ODA, in the backbone with more flexible ones in order to obtain a high performance melt processable PI. It is expected that the combination of flexible bonds, unsymmetrical moieties in the same macromolecule would yield new PIs offering a compromise between processability, on one side, and the physical and thermal characteristics, on the other. More-

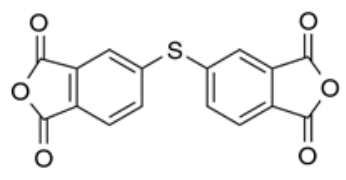

4,4'-TDPA

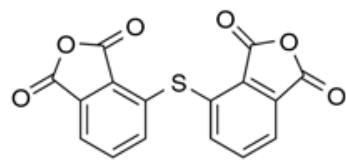

3,3'-TDPA

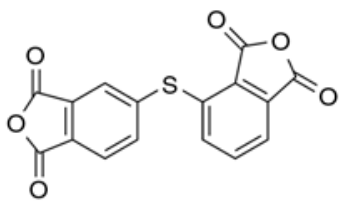

3,4'-TDPA

Figure 1. The structures of thioetherdiphthalic anhydride isomers 
over, to tailor a PI to satisfy specific requirements between processability and high $T_{g}$, a careful examination and selection of variety and composition of diamines is of great importance. Therefore, the first part of this work is to establish the basic structureproperty relationships in homopolyimides derived from mixed-TDPA and a variety of aromatic diamines. On the basis of $T_{g} \mathrm{~s}$, thermal stability, and rheological properties, some promising combination of diamines were selected to synthesized meltprocessable copolyimides afterwards. And the characterization was focused on processability of these copolyimides systems.

\section{Experimental}

\subsection{Monomer and other chemicals}

3- and 4-chlorophthalic anhydrides were obtained from Harbin Shidai Science and Technology Co., Ltd., China and used as received. Anhydrous sodium sulfide was purchased from Inner Mongolia Lichuan Chemistry Co., Ltd., China and used as received. Phthalic anhydride (PA) was purified by sublimation before use. 4,4'-ODA, mp 189 $190^{\circ} \mathrm{C}$, was purchased from Sinopharm Chemical Reagent Co., Ltd., China and used as received. 3,4'-ODA, mp $74 \sim 75^{\circ} \mathrm{C}$, was purchased from Changzhou Sunlight Medical Raw Material Co., Ltd., China and recrystallized from a 9/1 ratio of toluene/ethanol before use. 1,4-Phenylenediamine (PDA), mp 138 $140^{\circ} \mathrm{C}$, was purchased from Sinopharm Chemical Reagent Co., Ltd., and recrystallized from ethyl acetate. $m$-PDA, $\mathrm{mp} 63 \sim 65^{\circ} \mathrm{C}$, was purchased from Aladdin and recrystallized from a 9/1 ratio of toluene/cyclohexane before use. 1,4-bis(4-aminophenoxy)benzene (TPEQ), mp $173^{\circ} \mathrm{C}$, and TPER, $\mathrm{mp} 116^{\circ} \mathrm{C}$, were purchased from Changzhou Sunlight Medical Raw Material Co., Ltd., and used as received. All of the other reagents were from the Sinopharm Chemical Reagent Co., Ltd. and were of analytical grade.

\subsection{Characterization}

The inherent viscosities $\left(\eta_{\text {inh }}\right)$ were determined at $30^{\circ} \mathrm{C}$ with an Ubbelohde viscometer and the concentration was $0.5 \mathrm{~g} \cdot \mathrm{dL}^{-1}$ in $m$-cresol. ${ }^{1} \mathrm{H} \mathrm{NMR}$ spectra were recorded on a Bruker AV/ANCE at $400 \mathrm{MHz}$ using DMSO- $d_{6}$ as solvent and tetramethylsilane as an internal standard. The differential scanning calorimetry (DSC) was conducted on a Perkin-Elmer Diamond DSC system at a heating rate of $20^{\circ} \mathrm{C} / \mathrm{min}$ under nitrogen atmosphere. The thermal stability of PIs was estimated using a Perkin-Elemer Diamond TG/DTA thermogravimetric analyzer at a heating rate of $10^{\circ} \mathrm{C} / \mathrm{min}$ in nitrogen or an air atmosphere. The tensile measurements were carried out on an Instron model 5567 tensile tester at a crosshead speed of $5 \mathrm{~mm} / \mathrm{min}$ at room temperature.

The rheological properties of PIs were performed on a rotational Physica MCR 301 rheometer in an air flow. The parallel plate diameter was $25 \mathrm{~mm}$ and the gap was set to about $1.0 \mathrm{~mm}$ in all the runs. Sample discs of $25 \mathrm{~mm}$ diameter and $1 \mathrm{~mm}$ thickness were prepared by press-molding of the powder-like PI at $80^{\circ} \mathrm{C}$ under high pressure. The complex viscosity $\left(\eta^{*}\right)$ of PIs was measured by scanning the temperature from 280 to $420^{\circ} \mathrm{C}$ at a ramp rate of $3^{\circ} \mathrm{C} / \mathrm{min}$ with a frequency of $1 \mathrm{~Hz}$ and a strain of $1 \%$. Data acquisitions began $30 \mathrm{~min}$ after sample mounting. Meanwhile, dynamic time sweep test was performed at $340^{\circ} \mathrm{C}$ to evaluate the melt stability of PIs. The $\eta^{*}$, storage elastic modulus $\left(G^{\prime}\right)$ and the loss elastic modulus $\left(G^{\prime \prime}\right)$ data were isothermally collected at $340^{\circ} \mathrm{C}$ with a frequency of $1 \mathrm{~Hz}$ and a strain of $1 \%$. Steady shear viscosity of PIs was obtained subjected to small amplitude oscillatory shear at $1 \%$ strain over a frequency range from 0.01 to $100 \mathrm{~s}^{-1}$.

\subsection{Monomer synthesis}

3,3'-TDPA and 4,4'-TDPA and the isomer mixtures, mixed-TDPA were synthesized as reported in the literature [21]. 3,4'-TDPA was synthesized by a method revealed by a Chinese patent [20].

The composition of isomers in mixed-TDPA was calculated according to integrals of ${ }^{1} \mathrm{H}$ NMR spectra of the mixture and three isomers. The detailed information was as presented in reference [19]. As a result, the content of each isomer in the mixture was determined as follows: $21 \%$ (3, $3^{\prime}$-TDPA), $18 \%$ (4,4'-TDPA), and 61\% (3,4'-TDPA).

\subsection{Polyimides synthesis}

All PIs were prepared through one-pot method in $m$-cresol as shown in Figure 2. The inherent viscosity of PIs was regulated in the range of 0.4 to 


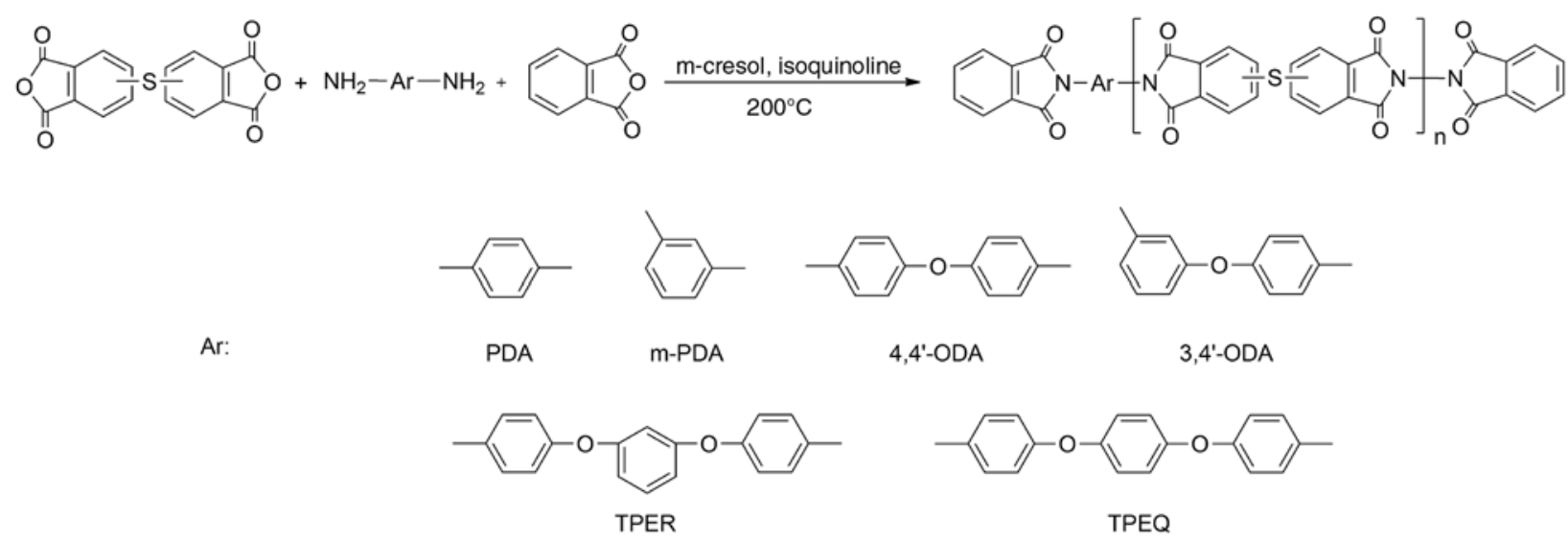

Figure 2. Synthesis of polyimides derived from mixed-TDPA via one-step polymerization

$0.5 \mathrm{dL} \cdot \mathrm{g}^{-1}$ by the stoichiometric imbalances between dianhydrides and diamines. PA as an endcapper was added for improving thermal stability of PIs. A typical procedure is described as follows as an example.

To a $100 \mathrm{ml}$ completely dried three-neck flask were added $2.0024 \mathrm{~g}$ (10.0 mmol) of 4,4'-ODA, $45 \mathrm{ml}$ $m$-cresol under nitrogen flow with stirring. After 4,4'-ODA was completely dissolved, $3.1975 \mathrm{~g}$ ( $9.8 \mathrm{mmol})$ of mixed-TDPA, $0.0592 \mathrm{~g}(0.4 \mathrm{mmol}$, designated as $2 \%$ ) of PA, and several drops of isoquinoline were added. The mixture was stirred at room temperature for a few minutes and then heated at $100^{\circ} \mathrm{C}$ for $1 \mathrm{~h}$ and $200^{\circ} \mathrm{C}$ for $8 \mathrm{~h}$. After cooling to room temperature, the viscous solution was diluted with additional $50 \mathrm{ml} \mathrm{m}$-cresol and then poured into $500 \mathrm{ml}$ of ethanol with stirring. The precipitate was filtered off, washed with boiling ethanol twice and dried in vacuo at $100^{\circ} \mathrm{C}$ for $10 \mathrm{~h}$.

The copolyimides based on mixed-TDPA and two different diamines were prepared by similar process as above. Mixed-TDPA was added in the $m$-cresol solution of two diamines until the diamines were completely dissolved.

\section{Results and discussion}

\subsection{Mixed-TDPA-based melt-processable homopolyimides}

\subsubsection{Basic properties of homopolyimides}

In order to appreciate the basic relationships between structure and properties of mixed-TDPA-based PIs, especially the melt processability, we synthesized a series of controlled molecular weight PIs from mixed-TDPA and a variety of diamines via onestep polymerization. Table 1 shows the basic prop-
Table 1. Properties of controlled molecular weight homopolyimides based on mixed-TDPA

\begin{tabular}{|c|c|c|c|c|c|}
\hline \multirow{2}{*}{ Diamines } & \multirow{2}{*}{$\begin{array}{c}\eta_{\text {inh }} \\
{\left[\mathrm{dL} \cdot \mathrm{g}^{-1}\right]}\end{array}$} & \multirow{2}{*}{$\begin{array}{c}\mathbf{T}_{\mathbf{g}} \\
{\left[{ }^{\circ} \mathbf{C}\right]}\end{array}$} & \multicolumn{2}{|c|}{$\mathbf{T}_{\mathbf{5} \%}\left[{ }^{\circ} \mathbf{C}\right]$} & \multirow{2}{*}{$\mathbf{R}_{\mathbf{w}^{\mathrm{a}}}$} \\
\hline & & & $\mathbf{N}_{2}$ & Air & \\
\hline $4,4^{\prime}$-ODA & 0.42 & 265 & 529 & 534 & $60 \%$ \\
\hline $3,4^{\prime}$-ODA & 0.45 & 246 & 527 & 538 & $60 \%$ \\
\hline PDA & 0.45 & 320 & 537 & 550 & $61 \%$ \\
\hline$m$-PDA & 0.41 & 289 & 524 & 530 & $62 \%$ \\
\hline TPEQ & 0.48 & 234 & 525 & 538 & $55 \%$ \\
\hline TPER & 0.47 & 217 & 529 & 518 & $58 \%$ \\
\hline
\end{tabular}

aResidual weight at $700^{\circ} \mathrm{C}$ in $\mathrm{N}_{2}$

erties of mixed-TDPA-based homopolyimides, each of which are designated by the abbreviation of diamines. The inherent viscosities of these PIs, which were controlled by the stoichiometric imbalances between dianhydrides and diamines, ranged from 0.4 to $0.5 \mathrm{dL} \cdot \mathrm{g}^{-1}$, indicating the polymer had similarly moderate molecular weights for comparing effectively while ensuring these PIs hold enough mechanical properties. According to our previous investigation, the value of $\eta_{\text {inh }}=0.36$ corresponds to the critical molecular weight for obtaining a flexible film from PIs based on mixedTDPA and ODA, below which the films become brittle. Meanwhile, all of the PIs in this range of molecular weight exhibit some extent of melt-flow behavior at elevated temperatures. PA was used as an endcapper for the high-temperature stability of the melt viscosity.

An important aspect of this study is the effect of the structural changes on the $T_{g}$. As presented in Table 1, the $T_{g}$ of mixed-TDPA-based PIs determined by DSC varies in a broad range from $217 \sim 320^{\circ} \mathrm{C}$ depending on the structures of the diamines. The introduction of flexible linkage diamines significantly decreases the $T_{g}$ such as TPER and TPEQ contrary to the effect of more 
rigid diamines such as PDA and $m$-PDA. As for the influence of isomerism, the results are as expected in terms of the effect of para/meta isomerism on the $T_{g} \mathrm{~s}$. PIs derived from para diamines show higher $T_{g}$ 's, i.e. $T_{g}=265^{\circ} \mathrm{C}$ (mixed-TDPA $/ 4,4^{\prime}$-ODA) $>246^{\circ} \mathrm{C}$ (mixed-TDPA $3,4^{\prime}$-ODA), $320^{\circ} \mathrm{C}$ (mixedTDPA/PDA $)>289^{\circ} \mathrm{C} \quad($ mixed-TDPA $/ m$-PDA $)$, $234^{\circ} \mathrm{C}$ (mixed-TDPA/TPEQ) $>217^{\circ} \mathrm{C}$ (mixedTDPA/TPER). This phenomenon can be attributed to the PIs with meta linkages disturb the intimate chain stacking necessary for intermolecular interaction [5]. As a result, the introduction of meta-linkages causes a significant decrease in $T_{g}$. These PI powders displayed no melting peak in the DSC scan, indicating that these polymers are totally amorphous. It is reasonable because the polyimides derived from three isomeric TDPA are amorphous as well [13]. In the case of mixed-TDPA-based PIs, the less ordered nature attributed by copolymerization of isomers suggests a greater degrees of molecular freedom and less intermolecular interaction.

Thermogravimetric analysis was performed to provide an insight into the thermal and thermoxidative stability of the PIs and to measure their decomposition temperatures. The temperatures at which a weight loss of $5 \%$ occurred both under $\mathrm{N}_{2}$ and air were recorded, along with the char yield at $700^{\circ} \mathrm{C}$ in $\mathrm{N}_{2}$. From the results in Table 1, mixed-TDPAbased PIs have a high thermal and thermoxidative stability $\left(T_{5} \%>520^{\circ} \mathrm{C}\right.$ both in $\mathrm{N}_{2}$ and air). It is worthwhile to note that the $T_{5 \%}$ of these copolyimides in air is higher than that in nitrogen which is just contrary to a majority of PIs. This phenomenon has been found in the other sulfur-containing polymers, which is ascribed to the different degradation mechanisms in various atmospheres $[12,22]$.

\subsubsection{Melt rheological properties of homopolyimides}

In order to obtain melt processable mixed-TDPAbased PIs, we investigate the relationships between melt viscosity and the structure of diamines. Molecular weight is one of the most important variables affecting the viscosity of a polymer melt. Therefore, all of the PIs were prepared at an established range of moderate molecular weight.

Similar to the variation of $T_{g}$ values with chemical structures, the more rigid structures tend to impart higher melt viscosities to the PIs. Within a series of
PIs based on a several diamines and the same level of molecular weight, melt viscosity generally increases in the order of increasing rigidity of the diamines.

The dynamic melt complex viscosity $\left(\eta^{*}\right)$ curves are shown in Figure 3 and the $\eta^{*}$ in three temperatures and minimum melt viscosity data are tabulated in Table 2. The melt viscosity of all the samples monotonically decreased with increasing temperature except the mixed-TDPA/PDA which has quite a high viscosity during the testing range, the curve of which was not presented in the figure. It is known that the asymmetric structure and meta linkages, such as the moieties in $3,4^{\prime}$-ODA and TPER, significantly decrease the degree of molecular packing and weaken the intermolecular force [17]. As a consequence, the melt flowability is improved by incorporation of these diamines. As presented in the table, the melt viscosity at three temperatures as well as the minimum viscosity of the PIs from TPER and 3,4'-ODA is much lower than that of 4,4'-ODA, which has similar melt viscosity in our previous research [19]. Take the $\eta^{*}$ of PI (4,4'-ODA) and PI (3,4'-ODA) as an example, the former has a $\eta^{*}$ of $21100 \mathrm{~Pa} \cdot \mathrm{s}$ when the processing temperature was $340^{\circ} \mathrm{C}$, and the minimum $\eta^{*}$

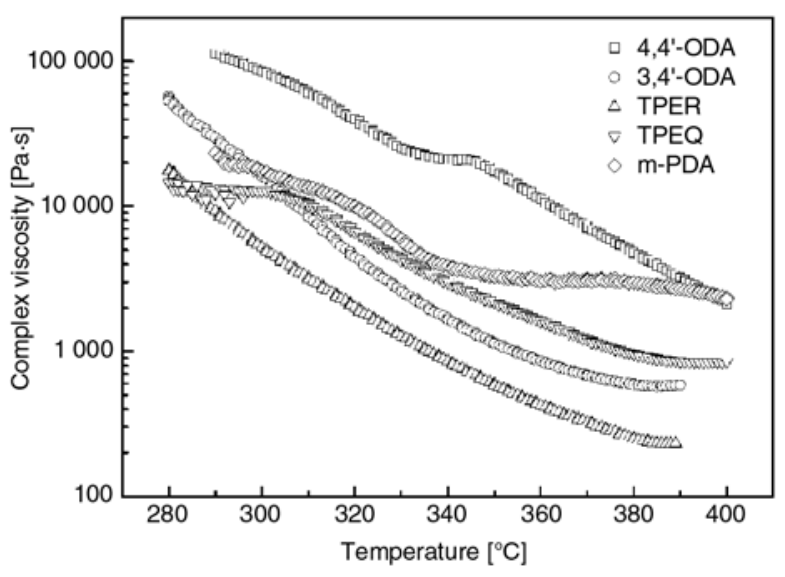

Figure 3. Melt complex viscosity of homopolyimides based on mixed-TDPA

Table 2. Melt complex viscosity of homopolyimides based on mixed-TDPA

\begin{tabular}{|l|c|c|c|r|}
\hline \multirow{2}{*}{ Diamines } & \multicolumn{2}{|c|}{ Melt complex viscosity $[\mathbf{P a} \cdot \mathbf{s}]$ at } & \multirow{2}{*}{$\begin{array}{c}\text { Min melt } \\
\text { viscosity }[\mathbf{P a} \cdot \mathbf{s}]\end{array}$} \\
\cline { 2 - 4 } & $\mathbf{3 2 0}^{\circ} \mathbf{C}$ & $\mathbf{3 4 0}^{\circ} \mathbf{C}$ & $\mathbf{3 8 0}^{\circ} \mathbf{C}$ & 2110 at $400^{\circ} \mathrm{C}$ \\
\hline $4,4^{\prime}-$ ODA & 39900 & 21100 & 4790 & 577 at $385^{\circ} \mathrm{C}$ \\
\hline $3,4^{\prime}-$-ODA & 4440 & 1700 & 595 & 231 at $387^{\circ} \mathrm{C}$ \\
\hline TPER & 2010 & 858 & 250 & 840 at $396^{\circ} \mathrm{C}$ \\
\hline TPEQ & 6650 & 2970 & 962 & 2290 at $400^{\circ} \mathrm{C}$ \\
\hline$m$-PDA & 9970 & 3890 & 2990 & 25000 at $341^{\circ} \mathrm{C}$ \\
\hline PDA & - & 25300 & 43000 & 250 \\
\hline
\end{tabular}


was approximately $2000 \mathrm{~Pa} \cdot \mathrm{s}$ at $400^{\circ} \mathrm{C}$. As for the PI (3,4'-ODA), the polymer has a $\eta^{*}$ of $1700 \mathrm{~Pa} \cdot \mathrm{s}$ at $340^{\circ} \mathrm{C}$ and a minimum $\eta^{*}$ of $577 \mathrm{~Pa} \cdot \mathrm{s}$ at $385^{\circ} \mathrm{C}$. Replacement of the rigid diamine with the more flexible ones causes a sharp decrease in the $\eta^{*}$. These results suggests that molecular interactions of the TPER and 3,4'-ODA based PIs are weaker than the other PI systems. In addition, the PI ( $m$ PDA) shows a lower melt viscosity than 4,4'-ODA. This phenomenon is valuable because the incorporation of $m$-PDA could impart a higher $T_{g}$ to the polymer than that of 4,4 '-ODA.

We try to elaborate the intrinsic disparity of melt flowability between PIs from different diamines by means of investigating the melt shear viscosity dependence on shear rate of PIs (4,4'-ODA and 3,4'-ODA) at several temperatures to demonstrate the effect of diamines. And the melt flow activation energy of polymer was calculated to compare between each other. The shear viscosity data as a function of shear rate for PIs from 4,4'-ODA and $3,4^{\prime}-\mathrm{ODA}$ at $330,340,350,360$, and $370^{\circ} \mathrm{C}$ are presented in Figure 4. Burks and St. Clair [10] had introduced sulfur in polyetherimides for the purpose of obtaining melt processable polyimides. According to the variation of apparent viscosity with shear rate, polythioetherimides in our system have shown better melt flowability than PIs based on 4,4'-bis(3,4-dicarboxyphenoxy)diphenylsulfide dianhydride. PIs from mixed-TDPA and 4,4'ODA, 3,4'-ODA show shear viscosity less than $10^{3} \mathrm{~Pa} \cdot \mathrm{s}$ when shear rate are close to $10^{2} \mathrm{~s}^{-1}$.

Simple mathematical models formulated for representing steady-flow viscosity data in terms of shear rate, the Carreau-Yasuda model has the following form (Equation (1)) [23]:

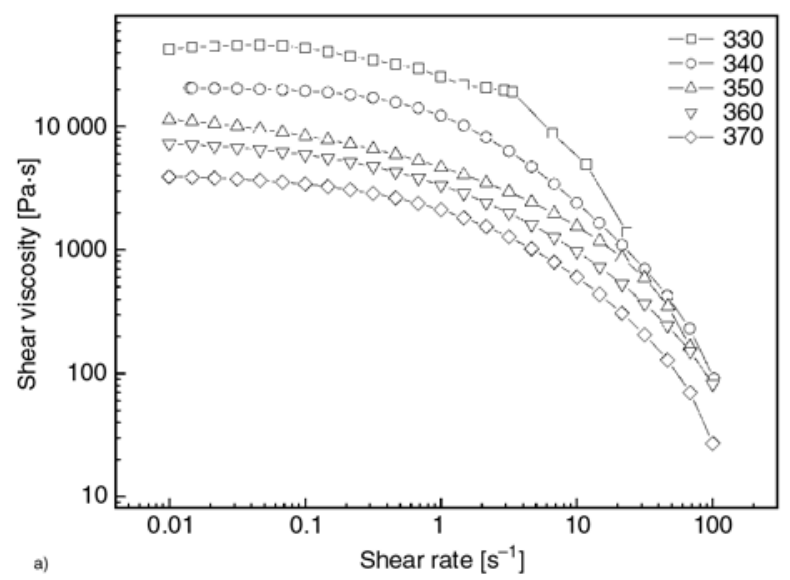

$\frac{\eta(\gamma)-\eta_{\infty}}{\eta_{0}-\eta_{\infty}}=\left[1+(\lambda \gamma)^{a}\right]^{\frac{n-1}{a}}$

where $\eta_{0}$ is called the zero-shear viscosity, $\eta_{\infty}$ is the infinite-shear-rate viscosity. $\lambda$ is a characteristic viscous relaxation time that defines the location of the transition from Newtonian to shear-thinning behavior. $a$ is a dimensionless parameter (sometimes called 'the Yasuda constant' since it was a parameter added to the Carreau equation by Yasuda) which describes the transition zone between the Newtonian plateau and the shear-thinning region and is inversely related to the breadth of this zone. The exponent $(n-1)$ defines the slope of the $\eta(\gamma)$ versus $\gamma$ curve within the power law region. For our purposes, $\eta_{\infty}$ is negligible (zero). Then the Carreau-Yasuda model was employed and the $\eta_{0}$ was calculated by the software of rheometer automatically. The continuous curves drawn in this figure are the Carreau-Yasuda equation fits of the experimental data. For each temperature, one can observe that the low shear rate viscosity plateau broadly spans to $10^{-2} \mathrm{~s}$, which allows the determination of the zero-shear-rate viscosity $\eta_{0}$ with satisfactory accuracy.

The values of $\eta_{0}$ are listed in Table 3 . The $\eta_{0}$ of PI $\left(3,4^{\prime}\right.$-ODA) are lower than that of PI (4,4'-ODA) at

Table 3. The zero-shear-rate viscosity of PI (4,4'-ODA) and PI (3,4'-ODA)

\begin{tabular}{|c|c|c|}
\hline \multirow{2}{*}{$\mathbf{T}\left[{ }^{\circ} \mathbf{C}\right]$} & \multicolumn{2}{|c|}{$\boldsymbol{\eta}_{\mathbf{0}}[\mathbf{P a} \cdot \mathbf{s}]$} \\
\hline 330 & $\mathbf{4 , 4}$-ODA & $\mathbf{3 , 4}$ '-ODA \\
\hline 340 & 44813 & 20343 \\
\hline 350 & 20985 & 10083 \\
\hline 360 & 15106 & 6900 \\
\hline 370 & 8013 & 6328 \\
\hline
\end{tabular}

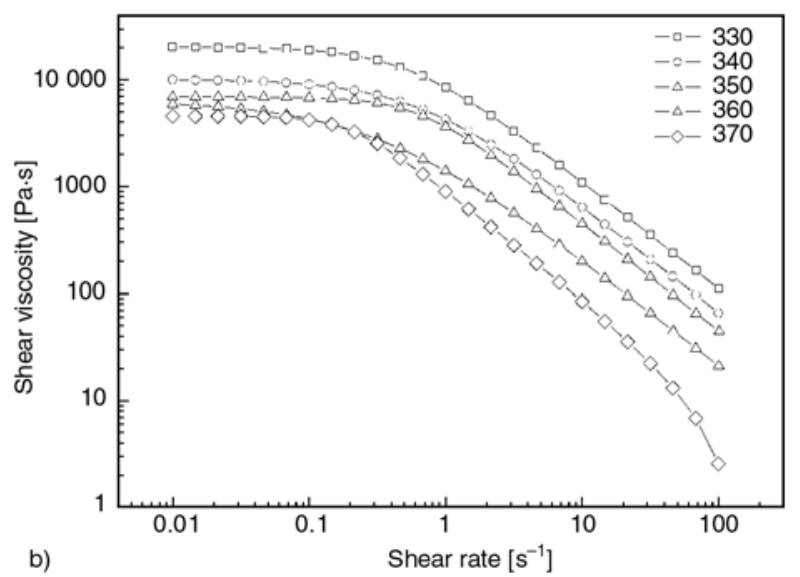

b)

Figure 4. Shear viscosity as a function of shear rate at different temperatures (a): PI(4,4'-ODA), (b): PI(3,4'-ODA) 
each temperature, indicating that the former exhibits higher melt flowability. The temperature dependence of the melt shear viscosity of polymer melts is one of the most important parameters in polymer flow, which can be expressed by the Arrhenius expression (Equation (2)) form [24]:

$\eta_{0}=A \exp \frac{E_{a}}{R T}$

where $R$ is the gas constant, $A$ is a constant, and $E_{a}$ is flow activation energy. $E_{a}$ varies widely from polymer to polymer, and depends on chain composition. In our system, PIs possess different chain flexibility values related to the diamine structure. This disparity in flexibility and melt flowability are reflected in the different values of $E_{a}$.

Values of $E_{a}$ are calculated by using $\eta_{0}$ data at temperatures from 330 to $370^{\circ} \mathrm{C}$. Thus, the $E_{a}$ values decrease from 178 to $118 \mathrm{~kJ} / \mathrm{mol}$, for the PI $\left(4,4^{\prime}\right.$ ODA) and PI (3,4'-ODA), respectively. And there is no doubt that this decrease is induced by the incorporation of asymmetrical moiety into polymer. Increasing flexibility by introduction of flexible bonds and unsymmetrical groups in PIs creates a lower energy barrier for the movement of the molecular chains in polymer melts. As a consequence, this modification leads to a reduction of $E_{a}$ demonstrating that a more flowable structure is obtained.

\subsection{Mixed-TDPA-based melt-processable copolyimides}

\subsection{1. $T_{g}$ of copolyimides}

It has been known that copolymerization is a simple and effective way for structure modification to balance desired properties, such as melt processability, thermal stability and mechanical properties [14]. Therefore, a series of copolyimides based on mixed-TDPA were prepared on the basis of the structure-property relationships for the homopoly- imides with the purpose of obtaining PIs with both nice melt-processability and a relative higher $T_{g} \mathrm{~s}$. Table 4 summarizes the properties of copolyimides based on mixed-TDPA and several compositions of diamines. The DSC scans of the copolyimides exhibited only one $T_{g}$, indicating that the various repeating units were randomly distributed along the polymer chain, thus a random copolymer structure had been formed. The $T_{g}$ s of these copolyimides were found to be in the range $229 \sim 255^{\circ} \mathrm{C}$. The Fox equation is usually used to estimate $T_{g}$ depending on composition for miscible blends and random copolymers in a bulk state [25]. In this study, the Fox equation was used to estimate composition dependence of the $T_{g} \mathrm{~S}$ of copolyimides. The constructed Equation (3) is as follows:

$$
\frac{1}{T_{g}^{*}}=\frac{w_{1}}{T_{g 1}}+\frac{w_{2}}{T_{g 2}}
$$

where $w_{1}$ and $w_{2}\left(w_{1}+w_{2}=1\right)$ are the weight fractions of diamine components, $T_{g 1}$ and $T_{g 2}$ represent the $T_{g}$ of each homopolyimides. Thus $T_{g}^{*}$ is the predicted glass transition temperature depending on the composition of the diamines and the results are listed in the table as well. Most of the $T_{g}^{*}$ values predicted by the Fox equation generally are very close to the actual $T_{g} \mathrm{~s}$ of the copolyimides. It is worth noting that the $T_{g}$ of PI ( $m$-PDA/TPER) is obviously higher than the predicted value $T_{g}^{*}$. This could be explained by the asymmetric structures of both these diamines significantly offered polymer a more twisted structure resulting that the movement of segment need more space and energy. However, the Fox equation could still be applied to estimate a $T_{g}$ value of copolyimides in our system.

\subsubsection{Melt rheological properties of copolyimides}

The dynamic melt complex viscosity curves of copolyimides are shown in Figure 5 and the com-

Table 4. Properties of copolyimides based on mixed-TDPA

\begin{tabular}{|l|c|c|c|c|c|c|}
\hline \multirow{2}{*}{ Diamines X/Y [mol ratio] } & \multirow{2}{*}{$\eta_{\text {inh }}\left[\mathbf{d L} \cdot \mathbf{g}^{-1}\right]$} & \multirow{2}{*}{$\mathbf{T}_{\mathbf{g}}\left[{ }^{\circ} \mathbf{C}\right]$} & \multirow{2}{*}{$\mathbf{T}_{\mathbf{g}}^{*}\left[{ }^{\circ} \mathbf{C}\right]$} & \multicolumn{2}{|c|}{$\left.\mathbf{T}_{\mathbf{5} \%}{ }^{\circ} \mathbf{C}\right]$} & \multirow{2}{*}{$\mathbf{R}_{\mathbf{w}}{ }^{\mathbf{a}}$} \\
\hline 3,4'/4,4'-ODA(1/1) & 0.48 & 255 & 255 & 532 & 539 & $59 \%$ \\
\hline TPER/TPEQ(1/1) & 0.47 & 229 & 226 & 521 & 525 & $56 \%$ \\
\hline TPER/3,4'-ODA(1/1) & 0.46 & 231 & 228 & 528 & 535 & $59 \%$ \\
\hline$m$-PDA/TPER(1/1) & 0.42 & 242 & 233 & 517 & 527 & $56 \%$ \\
\hline$m$-PDA/3,4'-ODA(3/7) & 0.44 & 255 & 253 & 520 & 529 & $59 \%$ \\
\hline PDA/3,4'-ODA(15/85) & 0.44 & 249 & 251 & 518 & 529 & $56 \%$ \\
\hline
\end{tabular}

aResidual weight at $700^{\circ} \mathrm{C}$ in $\mathrm{N}_{2}$ 
Table 5. Complex melt viscosity of copolyimides based on mixed-TDPA

\begin{tabular}{|c|c|c|c|c|}
\hline \multirow{2}{*}{ Diamines } & \multicolumn{3}{|c|}{ Complex melt viscosity $[\mathrm{Pa} \cdot \mathrm{s}]$ at } & \multirow{2}{*}{ Min melt viscosity $[\mathrm{Pa} \cdot \mathrm{s}]$} \\
\hline & $320^{\circ} \mathrm{C}$ & $340^{\circ} \mathrm{C}$ & $380^{\circ} \mathrm{C}$ & \\
\hline TPER/TPEQ(1/1) & 4020 & 2220 & 1270 & 1260 at $385^{\circ} \mathrm{C}$ \\
\hline TPER/3,4'-ODA(1/1) & 5440 & 2620 & 980 & 909 at $389^{\circ} \mathrm{C}$ \\
\hline $4,4^{\prime} / 3,4^{\prime}-\mathrm{ODA}(1 / 1)$ & 10900 & 8100 & 2580 & 1560 at $403^{\circ} \mathrm{C}$ \\
\hline$m$-PDA/TPER(1/1) & 7090 & 4100 & 999 & 745 at $395^{\circ} \mathrm{C}$ \\
\hline$m$-PDA/3,4'-ODA $(3 / 7)$ & 15200 & 5190 & 800 & 410 at $403^{\circ} \mathrm{C}$ \\
\hline $\mathrm{PDA} / 3,4^{\prime}-\mathrm{ODA}(15 / 85)$ & 13200 & 7420 & 1800 & 1190 at $400^{\circ} \mathrm{C}$ \\
\hline
\end{tabular}

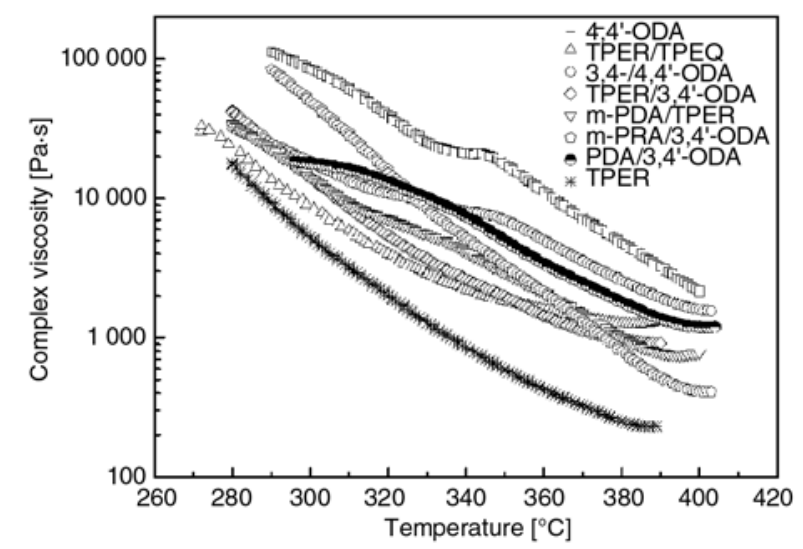

Figure 5. Melt complex viscosity of copolyimides based on mixed-TDPA

plex viscosity in three temperatures and minimum melt viscosity data are tabulated in Table 5. From the figure, most of the copolyimides exhibit enhanced melt flowability compared to PI (4,4'ODA) which has melt viscosity around $2000 \mathrm{~Pa} \cdot \mathrm{s}$ at $400^{\circ} \mathrm{C}$. In our previous research [19], PIs based on mixed-TDPA and 4,4'-ODA have a similar melt viscosity when the inherent viscosity was between the range of $0.4 \sim 0.5 \mathrm{dL} / \mathrm{g}$. However, the $T_{g} \mathrm{~s}$ of copolyimides decrease to a certain extent owing to the incorporation of flexible structures. Among the mixed-TDPA-based copolyimides, PI ( $m$-PDA/ TPER) and PI ( $m$-PDA/3,4'-ODA) simultaneously satisfied the demands of good melt flowability and less sacrifice of $T_{g} \mathrm{~s}$. Therefore, it has been demonstrated that processability of PIs as derived from mixed-TDPA can be be regulated in terms of diamine variety and composition. From the variations of $T_{g}$ s we can also figure out that flexible diamine with a relatively high molecular weight, namely a greater weight fraction in the composition, has a prominent effect on the physical properties of PIs.

\subsection{Melt stability of homo- and co-polyimides based on mixed-TDPA}

Isothermal viscosity measurements at $340^{\circ} \mathrm{C}$ were also conducted on most of the PIs to evaluate the melt stability in air. The curves of melt viscosity and data were showed in Figure 6 and Table 6, respectively. The melt viscosity ratio $(M V R)$ calculated by Equation (4) was usually employed to describe the melt stability [26]:

$M V R=\frac{M V 30}{M V 5}$

where $M V 5$ and $M V 30$ are the melt viscosities [Pa.s] measured at 5 and $30 \mathrm{~min}$, respectively, after the solid sample was completely molten. The polymer
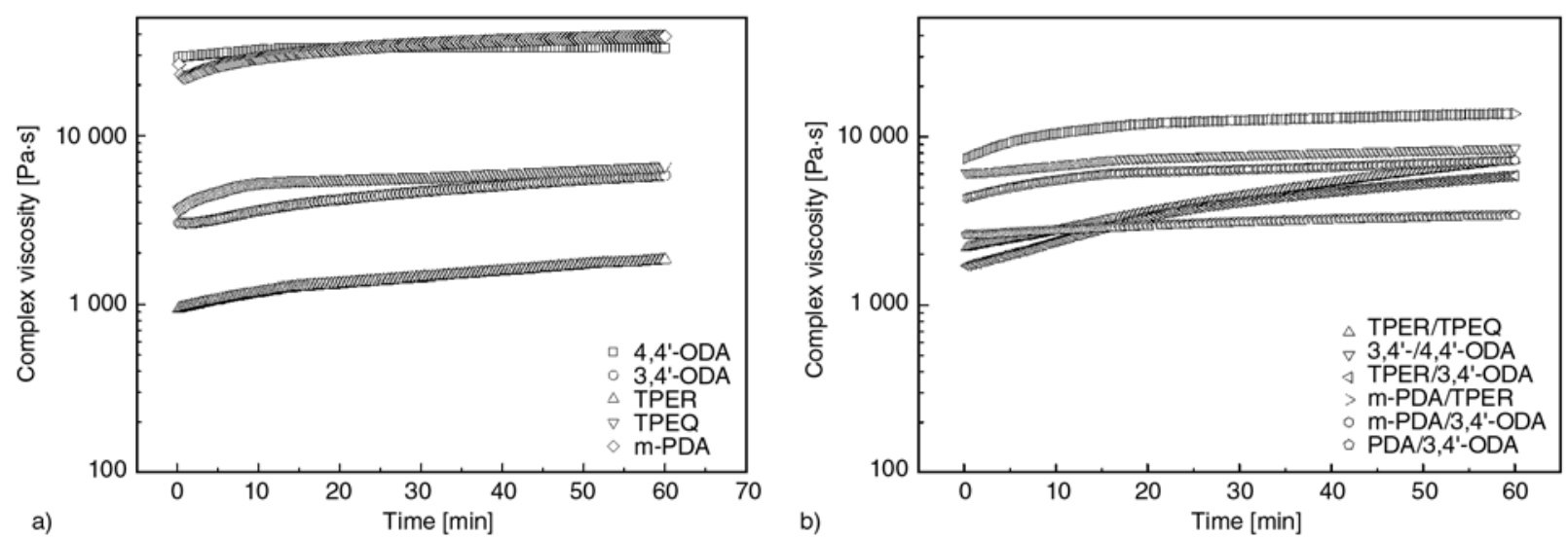

Figure 6. Complex viscosity of homopolyimides (a) and copolyimides (b) in air as a function of time at $340^{\circ} \mathrm{C}$ 
Table 6. Melt stability of homo- and copolyimides based on mixed-TDPA

\begin{tabular}{|l|r|r|r|c|}
\hline \multirow{2}{*}{\multicolumn{1}{c|}{ Diamines }} & \multicolumn{3}{|c|}{$\begin{array}{c}\text { Complex melt viscosity } \\
\text { [Pa·s] at }\end{array}$} & \multirow{2}{*}{ MVR } \\
\cline { 2 - 4 } & $\mathbf{5}$ min & $\mathbf{3 0}$ min & $\mathbf{6 0 ~} \mathbf{~ m i n}$ & \\
\hline $4,4^{\prime}$-ODA & 30800 & 33500 & 33200 & 1.1 \\
\hline $3,4^{\prime}$-ODA & 3190 & 4640 & 5800 & 1.4 \\
\hline TPER & 1060 & 1430 & 1830 & 1.3 \\
\hline TPEQ & 4600 & 5690 & 6600 & 1.2 \\
\hline$m$-PDA & 26000 & 35100 & 38900 & 1.3 \\
\hline TPER/TPEQ $(1 / 1)$ & 2450 & 4410 & 7220 & 1.8 \\
\hline TPER/3,4'-ODA $(1 / 1)$ & 2000 & 4090 & 5850 & 2.0 \\
\hline $4,4^{\prime} / 3,4^{\prime}-$-ODA $(1 / 1)$ & 6370 & 7780 & 8560 & 1.2 \\
\hline$m$-PDA/TPER $(1 / 1)$ & 9170 & 12500 & 13700 & 1.3 \\
\hline$m$-PDA/3,4'-ODA $(3 / 7)$ & 4970 & 6360 & 7240 & 1.3 \\
\hline PDA/3,4'-ODA(15/85) & 2670 & 3100 & 3410 & 1.2 \\
\hline
\end{tabular}

could be considered as having good melt stability if its $M V R$ value was located in the range of 1.0 1.5. In the experiment, the test specimen was loaded when the chamber was preheated, and the measurement was started when the temperature of the specimen and chamber was stabilized at $340^{\circ} \mathrm{C}$.

The isothermal viscosity data demonstrate that the viscosity increase after $60 \mathrm{~min}$ hold periods is acceptable and most of their $M V R$ are below 1.5. This indicates that undesirable crosslinking occurred slightly during the heating process which is favorable for melt-processing.

\subsection{Tensile properties of homo- and co-polyimides based on mixed-TDPA}

All PIs formed flexible films by dissolving polymer powder in $m$-cresol solution followed by volatizing the solvent in a forced air oven. The tensile properties of the thin films $(20 \sim 30 \mu \mathrm{m})$ were measured at room temperature, and are summarized in Table 7. As shown in the table, the tensile properties of PIs derived from mixed-TDPA indicated strengths at break of $100 \sim 120 \mathrm{MPa}$, tensile moduli ranging from 2600 to $3500 \mathrm{MPa}$, with an elongation at break of $4 \sim 8 \%$. As mentioned above, PIs with molecular weights in this range have nice mechanical properties as thermoplastic engineering materials. It is worthy to note that in the homopolyimides derived from mixed-TDPA, the meta-diaminebased PIs showed a higher tensile modulus than the para-diamine-based PIs. A similar behavior has been observed in PIs from isomeric biphenyltetracarboxylic dianhydrides (BPDA). The a-BPDA/ meta-diamine-based PIs showed a higher storage
Table 7. Tensile properties of homo- and copolyimides based on mixed-TDPA

\begin{tabular}{|l|c|c|c|}
\hline \multirow{2}{*}{\multicolumn{1}{|c|}{ Diamines }} & \multicolumn{3}{|c|}{ Tensile properties } \\
\cline { 2 - 4 } & $\begin{array}{c}\text { Strength } \\
{[\mathbf{M P a}]}\end{array}$ & $\begin{array}{c}\text { Modulus } \\
{[\mathbf{M P a}]}\end{array}$ & $\begin{array}{c}\text { Elongation } \\
{[\%]}\end{array}$ \\
\hline $4,4^{\prime}-$-ODA & 116 & 3200 & 6.4 \\
\hline $3,4^{\prime}-$-ODA & 120 & 3305 & 5.1 \\
\hline PDA & 121 & 3521 & 7.9 \\
\hline$m$-PDA & 111 & 3655 & 8.4 \\
\hline TPEQ & 101 & 2623 & 6.8 \\
\hline TPER & 116 & 3046 & 6.6 \\
\hline $4,4^{\prime}-$ ODA/3,4'-ODA(1/1) & 113 & 3025 & 6.1 \\
\hline TPEQ/TPER(1/1) & 110 & 2997 & 5.8 \\
\hline TPER/3,4'-ODA(1/1) & 107 & 3012 & 5.0 \\
\hline$m$-PDA/TPER(1/1) & 108 & 3140 & 4.3 \\
\hline$m$-PDA/3,4'-ODA(3/7) & 121 & 3435 & 7.4 \\
\hline PDA/3,4'-ODA(15/85) & 122 & 3174 & 8.6 \\
\hline
\end{tabular}

modulus measured by DMA as well as a higher tensile modulus. This phenomenon may be explained by a mechanism that a segment containing the meta-linkages can take a more extended conformation form than that containing the para-linkages [7-8]. However, this tendency is hard to distinguish in copolyimides based on mixed-TDPA, which may be attributed to that additional monomers disrupt the conformational consistency resulting in the loss of tensile modulus.

\subsection{Solubility}

Solubility is a major indicator for the intermolecular interaction, chain flexibility and conformation of PIs. Solubility of all the copolyimides were measured quantitatively by dissolving $0.1 \mathrm{~g}$ polymer powder in $2 \mathrm{ml}$ solvents and the results are summarized in Table 8. In general, the more bent and non-coplanar structure of molecular backbone result in weaker intermolecular interaction. Thus the PIs derived from $m$-PDA and 3,4'-ODA are more soluble than their counterparts from PDA and 4,4'-ODA. However, PIs from TPER and TPEQ show similarly nice solubility because of their flexible nature. Copolymerization is an effective method to enhance the solubility of insoluble PIs since the addition of a new component disrupts the symmetry and recurrence regularity of the polymer chain. It is also verified by investigating the solubility of copolyimides from diamines resulting better and less soluble PIs, such as $m$-PDA/3,4'-ODA, $m$-PDA/TPER, and PDA/3,4'-ODA. From the results of table, these copolyimides show improved 
Table 8. Solubility of homo- and copolyimides based on mixed-TDPA

\begin{tabular}{|c|c|c|c|c|c|c|}
\hline Diamines & DMAC & DMF & DMSO & NMP & m-cresol & $\mathrm{CH}_{3} \mathrm{Cl}$ \\
\hline PDA & - & - & - & +- & + & - \\
\hline$m$-PDA & - & - & +- & +- & + & - \\
\hline 3,4'-ODA & ++ & +- & ++ & ++ & ++ & ++ \\
\hline 4,4'-ODA & +- & +- & +- & +- & + & ++ \\
\hline TPEQ & ++ & ++ & ++ & ++ & ++ & ++ \\
\hline TPER & ++ & ++ & ++ & ++ & ++ & ++ \\
\hline$m$-PDA/3,4'-ODA & ++ & ++ & + & ++ & ++ & +- \\
\hline$m$-PDA/TPER & ++ & +- & + & ++ & ++ & - \\
\hline PDA/3,4'-ODA & ++ & ++ & ++ & ++ & ++ & ++ \\
\hline
\end{tabular}

++ : soluble at room temperature; + : soluble on heating; +- : partially soluble on heating; -: insoluble

solubility than the homopolyimides based on mPDA and PDA, respectively.

\section{Conclusions}

A series of PIs based on mixed-TDPAs have been synthesized with a variety of aromatic diamines. According to the basic structure-property relationships several promising melt processable copolyimides combined with melt flowability and relatively higher $T_{g}$ were developed. The copolyimides with controlled molecular weights were characterized by good mechanical properties, lower melt viscosities compared to the PI (mixed-TDPA/ 4,4'-ODA) while remaining high thermal stability. The decrease of $E_{a}$ from $178 \mathrm{~kJ} / \mathrm{mol}$ of PI (4,4'ODA) to $118 \mathrm{~kJ} / \mathrm{mol}$ of PI (3,4'-ODA) demonstrated that the incorporation of unsymmetrical structure into the polyimide backbone enhances the melt flowability. From the melt thermal stability point of view, this series of copolyimides are favorable to be applied as thermoplastic engineering materials.

\section{Acknowledgements}

We are grateful for the financial support from the Qian Jiang Talent Program of Zhejiang Province (2007R10021), Natural Science Foundation of Ningbo City (2009A610055) and International Science and Technology Cooperation Program of Ningbo City (2009D10017), China.

\section{References}

[1] Wilson D., Stenzenberger H. D., Hergenrother P. M.: Polyimides. Blackie and Son, Glasgow (1990).
[2] Wang K., Fan L., Liu J-G., Zhan M-S., Yang S-Y.: Preparation and properties of melt-processable polyimides based on fluorinated aromatic diamines and aromatic dianhydrides. Journal of Applied Polymer Science, 107, 2126-2135 (2008).

DOI: $10.1002 / a p p .25056$

[3] Zuo H. J., Chen J. S., Yang H. X., Hu A. J., Fan L., Yang S. Y.: Synthesis and characterization of meltprocessable polyimides derived from 1,4-bis(4-amino2-trifluoromethylphenoxy)benzene. Journal of Applied Polymer Science, 107, 755-765 (2008).

DOI: $10.1002 /$ app. 26965

[4] Li Q., Fang X., Wang Z., Gao L., Ding M.: Polyimides from isomeric oxydiphthalic anhydrides. Journal of Polymer Science Part A: Polymer Chemistry, 41, 3249-3260 (2003).

DOI: $10.1002 /$ pola.10918

[5] Shi Z., Hasegawa M., Shindo Y., Yokata R., He F., Yamaguchi H., Ozawa H.: Thermo-processable polyimides with high thermo-oxidative as derived from oxydiphthalic anhydride and bisphenol A type dianhydride. High Performance Polymers, 12, 377-393 (2000). DOI: $10.1088 / 0954-0083 / 12 / 3 / 302$

[6] Ding M.: Isomeric polyimides. Progress in Polymer Science, 32, 623-668 (2007).

DOI: 10.1016/j.progpolymsci.2007.01.007

[7] Hergenrother P. M., Watson K. A., Smith J. G., Connell J. W., Yokota R.: Polyimides from 2,3,3',4'biphenyltetracarboxylic dianhydride and aromatic diamines. Polymer, 43, 5077-5093 (2002).

DOI: $10.1016 / \mathrm{S} 0032-3861(02) 00362-2$

[8] Chen C., Yokota R., Hasegawa M., Kochi M., Horie K., Hergenrother P.: Isomeric biphenyl polyimides. (I) Chemical structure-property relationships. High Performance Polymers, 17, 317-333 (2005). DOI: $\underline{10.1177 / 0954008305055556}$

[9] Fang X. Z., Wang Z., Yang Z. H., Gao L. X., Li Q. X., Ding M. X.: Novel polyimides derived from 2,3,3',4'benzophenonetetracarboxylic dianhydride. Polymer, 44, 2641-2646 (2003). DOI: $\underline{10.1016 / \mathrm{S} 0032-3861(03) 00181-2}$ 
[10] Burks H. D., St. Clair T. L.: Processable aromatic polyimides. Journal of Applied Polymer Science, 30, 2401-2411 (1985).

DOI: $10.1002 /$ app.1985.070300611

[11] Glatz F. P., Mülhaupt R.: Syntheses and properties of soluble poly(arylene thioether imide)s and the corresponding poly(arylene sulfone imide)s. Polymer Bulletin, 31, 137-143 (1993).

DOI: $10.1007 / \mathrm{BF} 00329958$

[12] Liu J-G., Shibasaki Y., Ando S., Ueda M.: Synthesis and thermal properties of polythioetherimides derived from 4,4'-[p-thiobis(phenylenesulfanyl)]diphthalic anhydride and various aromatic diamines. High Performance Polymers, 20, 221-237 (2008). DOI: $\underline{10.1177 / 0954008307082508}$

[13] Zhang M., Wang Z., Gao L. X., Ding M. X.: Polyimides from isomeric diphenylthioether dianhydrides. Journal of Polymer Science Part A: Polymer Chemistry, 44, 959-967 (2006). DOI: $10.1002 /$ pola.21204

[14] de Abajo J., de la Campa J. G.: Processable aromatic polyimides. Progress in Polyimide Chemistry I: Advances in Polymer Science, 140, 23-59 (1999). DOI: $10.1007 / 3-540-49815-X 2$

[15] Hasegawa M., Sensui N., Shindo Y., Yokota R.: Improvement of thermoplasticity for s-BPDA/PDA by copolymerization and blend with novel asymmetric BPDA-based polyimides. Journal of Polymer Science Part B: Polymer Physics, 37, 2499-2511 (1999). DOI: 10.1002/(SICI)1099-0488(19990901)37:17 $\leq 2499:$ AID-POLB21>3.0.CO;2-G

[16] Yokota R., Yamamoto S., Yano S., Sawaguchi T., Hasegawa M., Yamaguchi H., Ozawa H., Sato R.: Molecular design of heat resistant polyimides having excellent processability and high glass transition temperature. High Performance Polymers, 13, S61-S72 (2001).

DOI: $\underline{10.1088 / 0954-0083 / 13 / 2 / 306}$
[17] Simone C. D., Scola D. A.: Phenylethynyl end-capped polyimides derived from 4,4'-(2,2,2-trifluoro-1phenylethylidene)diphthalic anhydride, $4,4^{\prime}$-(hexafluoroisopropylidene)diphthalic anhydride, and 3,3',4,4'biphenylene dianhydride: Structure-viscosity relationship. Macromolecules, 36, 6780--6790 (2003).

DOI: $10.1021 / \mathrm{ma} 0300701$

[18] Tamai S., Oikawa H., Ohta M., Yamaguchi A.: Melt processable copolyimides based on 4,4'-bis(3aminophenoxy)biphenyl. Polymer, 39, 1945-1949 (1998).

DOI: $\underline{10.1016 / \mathrm{S} 0032-3861(97) 00491-6}$

[19] Han Y., Fang X. Z., Zuo X. X.: The influence of molecular weight on properties of melt-processable copolyimides derived from thioetherdiphthalic anhydride isomers. Journal of Materials Science, 45, 1921-1929 (2010).

DOI: $10.1007 / \mathrm{s} 10853-009-4179-\mathrm{Z}$

[20] Yang Z. H., Ding M. X.: The method of synthesis of diphenylthioethertetracarboxylic acid and its derivatives. Chinese Patent 1081436A (1994).

[21] Evans T. L., Williams F. J., Donahue P. E., Grade M. M.: Synthesis and polymerization of thioether dianhydride. Polymer Preprints, 24, 268-269 (1984).

[22] Li X-G., Huang M-R., Bai H., Yang Y-L.: High-resolution thermogravimetry of polyphenylene sulfide film under four atmospheres. Journal of Applied Polymer Science, 83, 2053-2059 (2002).

DOI: 10.1002/app.10011

[23] Bird R. B., Armstrong R. C., Hassager O.: Dynamics of polymeric liquids, fluid mechanics. Wiley, New York (1987).

[24] Graessley W. W., Raju V. R.: Some rheological properties of solutions and blends of hydrogenated polybutadiene. Journal of Polymer Science Polymer Symposia, 71, 77-93 (1984).

DOI: $10.1002 /$ polc. 5070710109

[25] Fox T. G.: Influence of diluent and of copolymer composition on the glass temperature of a polymer system. Bulletin of the American Physical Society, 1, 123-125 (1956).

[26] Kuroki T., Sakata Y., Okumura T.: Crystalline polyimide for melt molding with satisfactory thermal stability. U.S. Patent 6458912 (2002). 